\title{
Method to make accurate measurements of refractive index
}

\section{Carlos Robledo-Sánchez, Nazario Bautista-Elivar}

Carlos Robledo-Sánchez, Nazario Bautista-Elivar, "Method to make accurate measurements of refractive index," Proc. SPIE 9663, Eighth International Topical Meeting on Education and Training in Optics and Photonics, 96632L (6 October 2003); doi: 10.1117/12.2207471

SPIE Event: Eighth International Topical Meeting on Education and Training in Optics and Photonics, 2003, Tucson, Arizona, United States 


\title{
Method to make accurate measurements of refractive index
}

\author{
Carlos Robledo-Sánchez, Nazario Bautista-Elivar.
}

Benemérita Universidad Autónoma de Puebla, Facultad de Ciencias FísicoMatemáticas. Av. Sn. Claudio y Río Verde Col. Sn. Manuel, C.P. 72570 , Puebla, pue., México.

email: crobledo@fcfm.buap.mx ,nelivar@fcfm.buap.mx

We propose in this work the use a diffraction grating to calculate the wavelength inside a transparent material of parallel plane faces, with the purpose of measuring the magnitude of their refractive index. The errors of this method and the precision are discussed .

Keywords: metrology, diffraction grating, refractive index.

\section{Introduction}

One of the important parameters used in optics is the refractive index. There are different methods to measure the refractive index in the visible region. One of the techniques used to carry out these measurements uses the method of minimum deviation that suffers a ray of light when crossing a prism ${ }^{(1)}$. Other common methods are based on the determination of the critical angle and in the Brewster angle ${ }^{(2)}$. A method of determination of the refractive index using prisms was described recently by $\mathrm{Hsiu}^{(3)}$. In the method of Hsiu, the incident beam is normal to the first surface of the prism the refraction is manifested only in the second surface. The relationship obtained under this configuration is $n=\operatorname{sen}(\alpha+\delta) / \operatorname{sen} \alpha$, where $\alpha$ the angle of the prism and $\delta$ the deviation angle that suffers the beam in the second surface. The method of Hsiu can be used to determine refractive index of lens doing some modifications. Most of these methods are based on the application of the refraction law. The method that we propose in this paper considers the diffraction of the light.

\section{Theory}

The diffraction grating is normally used for measuring wavelength through the grating equation

$$
a \sin \theta_{m}=m \lambda
$$

\footnotetext{
Eighth International Topical Meeting on Education and Training in Optics and Photonics, edited by Barry L. Shoop, Grover Swartzlander Jr., Proc. of SPIE Vol. 9663, 96632L

(C) 2003 SPIE, OSA, ICO · doi: 10.1117/12.2207471
} 
for the case when the incident plane wavefronts of light make an angle of $0^{\circ}$ with the plane of the grating, $m$ is order of interference. The zero-order principal maximum provides no information about the wavelength of the illumination. Information about the wavelength of the illuminating light can only be obtained by measuring the angular position of the higher-order principal maximum. We will employ this method for measuring wavelength inside of a optic glass. It is known that a wave inside a transparent material changes its space period in the form ${ }^{(4)}$

$$
\lambda^{\prime}=\frac{\lambda}{n},
$$

this result is used to measure $n$ measuring the wavelength $\lambda^{\prime}$ inside the material and using $\lambda$ of a well-known source. Considering the Ec.(1) the separation $d y$ of two diffraction orders is

$$
d y=\lambda \frac{s}{a},
$$

where $s$ is the distance from the grating to observation screen, $a$ is the separation of slits. If the same experiment is realized inside a material with $n$ index, then the wavelength change by

$$
\lambda^{\prime}=d y^{\prime} \frac{a}{s},
$$

Finally using the Ec. (1) we can obtain $n$ calculating $\lambda / \lambda^{\prime}$; where $\lambda$ is the wavelength in the vacuum of a well-known source, $\lambda^{\prime}$ is the wavelength inside the material. Substituting the Ec. (4) and Ec. (5) into the Ec. (2) that $n=\lambda / \lambda^{\prime}$ can be obtained by

$$
n=d y / d y^{\prime}
$$

where $d y$ and $d y^{\prime}$ are the separation of diffraction orders when the experiment is realized in the vacuum and inside a material, respectively.

\section{Experimental procedure}

The experimental setup consist of a He-Ne laser of $3 \mathrm{~mW}$ and $632.8 \mathrm{~nm}$ and optical glass cube as material under test. The cube is mounted on a XY positioning stage platform. The parameter $a$ of the diffraction grating used is 6.81 microns. The measurements of $d y$ and $d y^{\prime}$ was carry out with a CCD camera. The measurements was doing placing the diffraction grating close to the DA face of the cube see Fig.1, measuring the separation $d y^{\prime}$ among -1 and +1 diffracted orders in the opposite face CD of the cube. Subsequently we measure $d y$ among the same orders but now outside of the 
cube. The pattern of diffraction inside the cube is observed in the $\mathrm{CB}$ face and outside in the BF face, see Fig. 1.

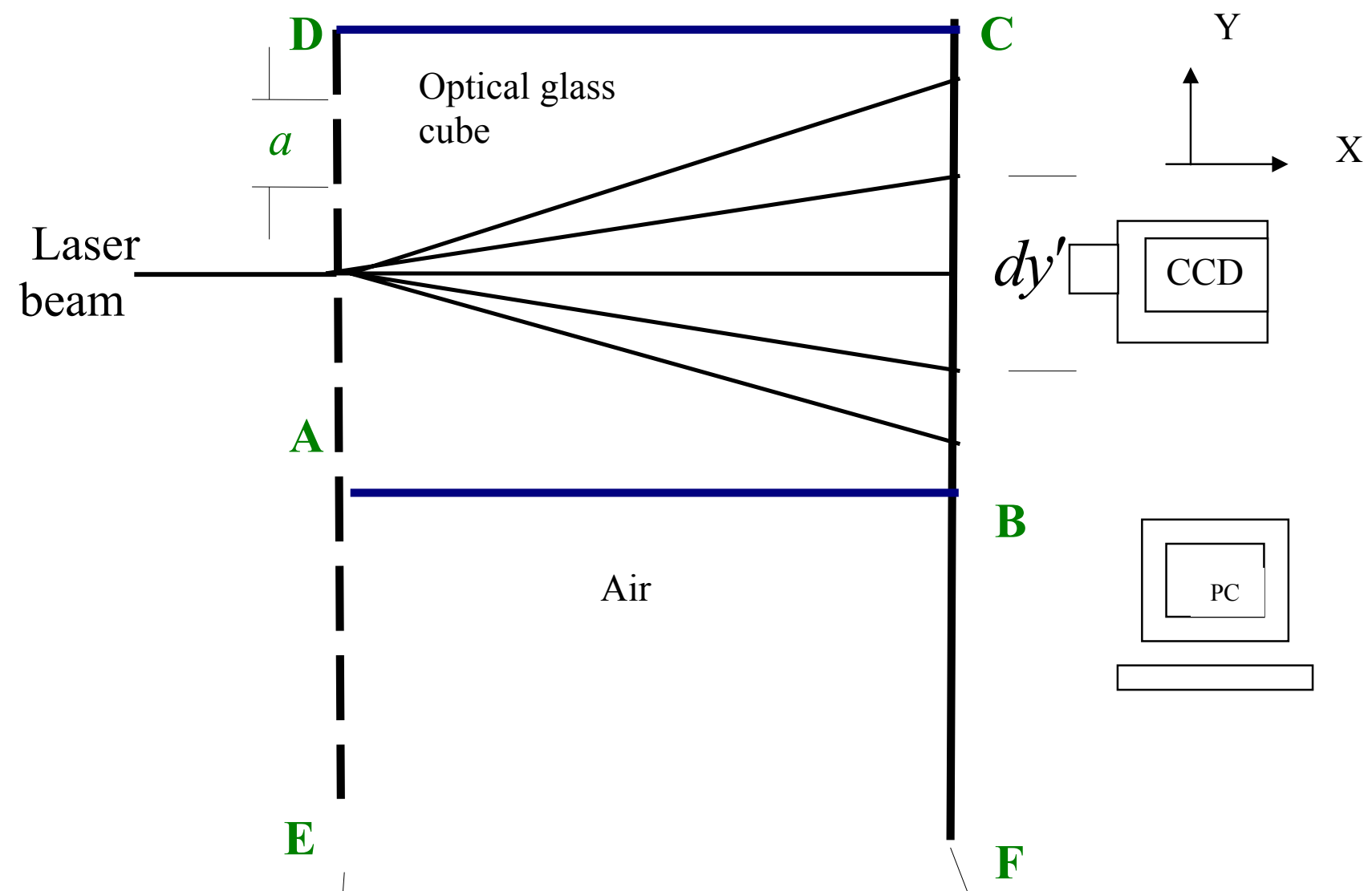

Diffraction grating Diffuser sheet

Fig. 1. Experimental setup to measuring the refraction index of a optical glass cube using diffraction.

To observe the diffraction grating we put a diffuser sheet in the $\mathrm{CB}$ face of the cube. To observe the diffraction pattern inside and outside of the cube this was translated laterally in the Y direction. The measurements was realize with a CCD camera with a zoom lens. The Fig. 2 show the diffraction patterns obtained. 


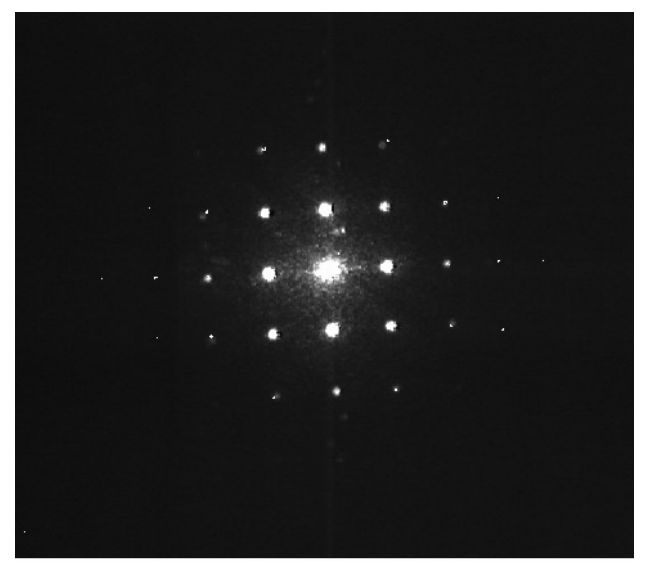

(a) Diffraction pattern inside the optical glass cube

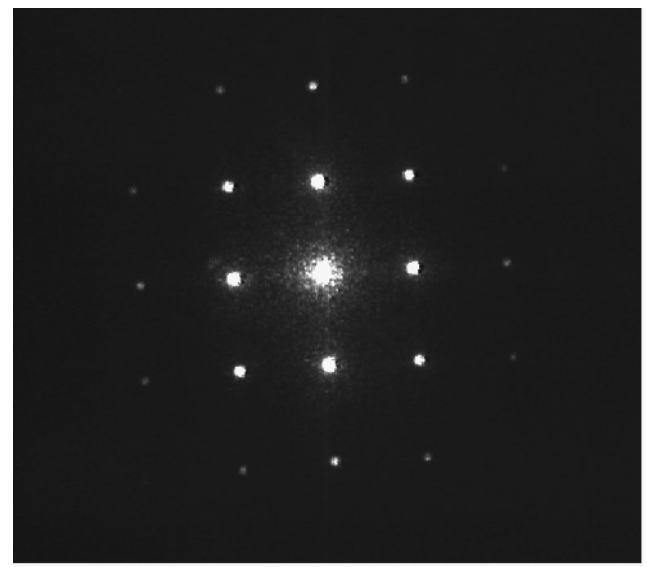

(b) Diffraction pattern in air

\section{Results}

Fig. 2. Diffraction patterns.

Using the images of Fig. 2 we obtained the value of $d y^{\prime}$ and $d y$ of 190.1 and 289 pixels, respectively. Giving the magnitude of 1.521 for the refractive index. The value specified of $n$ is 1.52504 for the He-Ne laser. Our result obtained has a precision of $0.2 \%$.

\section{Conclusions}

We used a diffraction experiment to calculate the refractive index of a glass cube. This method is simple and has good precision. This experiment can be use to show in the classroom in an elementary optics course the effect of how the wavelength change inside the materials. The method gives as an alternative technique of easy implementation in ours optics laboratories.

One of authors N. Bautista-Elivar thanks the support granted by CONACyT.

\section{References}

(1) R.S. Longhurst, "Geometrical and physical optics". Longmans, Green, New York london (1962), p.p. 72-73.

(2) C. Harvey Palmer, "Optics, experiments and demotrations", the Johns-Hopkins Press Baltimore (1962).

(3) H.Hsiu, "Accurate measurement of refractive index", Eng. Lab. Notes in opt. $\backslash \&$ phot. News s-4 (1993).

(4) P. Hariharan,"Optical interferometry", Academic Press, Inc. (1985). 